\title{
¿CÓMO JUSTFFICA DAVID RICARDO SU PROPUESTA DE LIBRE COMERCIO?
}

\section{HOW DOES DAVID RICARDO JUSTIFY HIS FREE TRADE PROPOSAL?}

\section{GONZALO JONÁS PAREDES REYES}

Universidad Católica de Santiago de Guayaquil. gonzalo.paredes01@cu.ucsg.edu.ec

RESUMEN

El objetivo es encontrar cuáles son los supuestos teóricos de la economía clásica, desde la perspectiva de David Ricardo, que permitan explicar la propuesta de libre comercio como vía para acrecentar de forma acelerada la riqueza de las naciones. Para llevar a cabo este análisis se pasa a revisión "Principles of PoliticalEconomy and Taxation", su más importante obra, buscando las principales afirmaciones para explicar cada supuesto de su modelo y articular las ideas que permitan exponer cómo Ricardo llega a su más notable recomendación a la economía mundial: la división internacional del trabajo. Se concluye que la confrontación entre productores y terratenientes se solucionaría con la merma de este último -a través de la eliminación de las barreras arancelarias-, que tiene un riesgo bajo al que asume el primero en la actividad económica. La eliminación de trabas al libre comercio llevaría a una expansión más acelerada de una economía.

PALABRAS CLAVE: renta, teoría, libre comercio, productores, terratenientes.
ABSTRACT The purpose of this study is to find out what the theoretical assumptions of classical economics are, from the perspective of David Ricardo, who explains the proposed free trade as a way of rapidly increasing wealth of nations. To perform this analysis, one of his most important works: "Principles of Political Economy and Taxation", has been reviewed, in searching the main statements to explain each course of his model and articulate ideas to show how Ricardo reaches its most notable recommendation for global economy: The international division of labor. We conclude that the struggle between producers and landowners would be solved with the decline of the latter-through the removal of all tariff barriers, which have a low risk that is assumed by the first in the economic activity. The elimination of obstacles to free trade would lead to a rapid expansion of the economy.

KEYWORDS: rent, theory, free trade, producers, landowners. 
INTRODUCCIÓN

El tema a tratar en este trabajo es la justificación del libre comercio que realiza David Ricardo en su obra principal "Principles of PoliticalEconomy and Taxation". En muchos de los estudios sobre el pensamiento económico de Ricardo y de sus aportes a la construcción de las ciencias económicas, se tratan por separado las ideas expuestas por este fundador de la economía clásica.

Sus ideas, que son parte de una construcción teórica muy bien elaborada para alguien de su época, sentaron las bases para una economía mundial acoplada a la División Internacional del Trabajo, expuesta por Smith en las Riquezas de las Naciones. Los manuales de economía internacional describen cómo opera la ventaja comparativa, pero no explican de dónde proviene el contexto en que se la diseña y los intereses de los actores involucrados.

La relevancia del presente trabajo está en que se articulan las principales ideas de David Ricardo, sin presentarlas de forma aislada las unas de las otras, para demostrar por qué el libre comercio es una condición para que aumente la riqueza de un país. ¿Cuál es la construcción teórica que está detrás? ¿Cuáles son los supuestos de tal construcción? ¿Dónde desemboca la concepción de renta diferencial? ¿Para qué la bosqueja? ¿Qué lugar ocupa la teoría Malthusiana? ¿Cuál es la influencia de Adam Smith para la obra de Ricardo?

Este aporte se ocupa en su totalidad de cómo Ricardo justifica el libre comercio en una economía mundial donde las asimetrías eran muy profundas. Para estudios posteriores queda por exponer cuáles son las limitaciones de su desarrollo teórico, y su inaplicabilidad para los actuales países en vías de desarrollo.

\section{LOS ANTECEDENTES DE LA VENTAJA COMPARATIVA}

Aunque Adam Smith cambió la forma de entender el comercio en sus contemporáneos, para los propios de Gran Bretaña les incomodaba, dado que los holandeses eran más eficientes en todos los productos que los que ellos producían. En esta condición, Holanda decidiría si comerciar o no, ya que ellos tendrían la ventaja absoluta en todas las mercancías.

Appleyard y Field (2003) reseñan que las ideas de Smith sobre la ventaja absoluta estaban determinadas cuando los factores eran perfectamente móviles y que la industria se asienta donde exista mayor ventaja absoluta. De igual forma, el trabajo y el capital se desplazan hacia el área donde la productividad y los rendimientos son más elevados.

Sin embargo, para Ricardo la historia es diferente internacionalmente. Aunque el comercio internacional puede operar con base en la ventaja absoluta (por ejemplo, el comercio entre las zonas tropical y templada), dada la inmovilidad internacional del factor de producción, las ganancias del comercio también pueden ser fruto de la ventaja comparativa. Ricardo presentó un caso en que describió la producción de dos bienes, vino y tela, en Inglaterra y Portugal:

TABLA 1. REPRESENTACIÓN DE LAS PRODUCTIVIDADES EN LA ELABORACIÓN DE VINO Y TELA

\begin{tabular}{lll} 
PAÍS & VINO & TELA \\
\hline PORTUGAL & 80 & 90 \\
\hline INGLATERRA & 120 & 100 \\
\hline
\end{tabular}

Fuente: Adaptado de Ricardo (1985).

Aún cuando Portugal produce ambos artículos a menores costos en días de trabajo, le conviene comerciar con Inglaterra si a cambio de una unidad de vino, que le cuesta 80 días, recibe una unidad de tela que le cuesta 90 días, ahorrándose dies días de trabajo. A Inglaterra le conviene también el cambio, porque por una unidad de tela que le cuesta cien días recibe una unidad de vino que internamente le cuesta 120 días, ahorrándose 20 días. Ricardo (1985) afirmó lo siguiente:

En un sistema de comercio libre, absolutamente libre, cada país invertirá naturalmente su capital y su trabajo en empleos tales que sean lo más beneficioso para ambos. Esta persecución del provecho individual está admirablemente relacionada con el bienestar universal. Distribuye el trabajo en la forma más efectiva y económica posible al estimular la industria, recompensar el ingenio y por el más eficaz empleo de las aptitudes peculiares con que lo ha dotado la naturaleza; al incrementar la masa general de la producción, difunde el beneficio general y une a la sociedad universal de las naciones en todo el mundo civilizado con un mismo lazo de interés e intercambio común a todas ellas. Es este principio el que determina que el vino se produzca en Francia y Portugal, que los cereales se cultiven en América y en Polonia, y que Inglaterra produzca artículos de ferretería y otros. (p.102)

Así, según Tugores (2006), la ventaja comparativa significa "que la especialización debe 
hacerse atendiendo a aquello que cada país (jugador) es capaz de hacer comparativamente mejor, es decir, analizando en qué actividad su ventaja es mayor o su desventaja menor" (p.12). Un país tiene ventaja comparativa en la producción de un bien si el costo de oportunidad en la producción de este bien en términos de otros bienes es inferior en este país de lo que lo es en otros países (Krugman y Obstfeld, 2006).

\section{LA DESCRIPCIÓN DEL MODELO DE LA TEORÍA RICARDIANA}

Los supuestos del modelo ricardiano son los siguientes (Appleyard y Field, 2003, p.27):

1. Cada país tiene una dotación fija de recursos, y todas las unidades de cada recurso particular son idénticas.

2. Los factores de producción son completamente móviles entre usos alternativos dentro de un país. Este supuesto implica que los precios de factores de producción también son iguales entre estos usos alternativos.

3. Los factores de producción son completamente inmóviles externamente; es decir, no se mueven entre países. Por consiguiente, los precios de los factores pueden ser diferentes entre países antes del comercio.

4. En el modelo se emplea una teoría del valor trabajo. Por tanto, el valor relativo de un bien está basado únicamente en su contenido de trabajo relativo. Desde el punto de vista de la producción, esto implica que: a) No se utilizan otros insumos en el proceso de producción, Otros insumos que puedan existir están medidos en relación con el trabajo incorporado en su producción, o c) La relación otros insumos/trabajo es igual en todas las industrias.

5. El nivel de tecnología es fijo en ambos países, aunque la tecnología puede diferir entre ellos.

6. Los costos unitarios de producción son constantes. Por tanto, las horas de trabajo por unidad de producción de un bien no cambian, independientemente de la cantidad producida. Esto significa que la curva de oferta de cualquier bien es horizontal.

7. Hay pleno empleo.

8. La economía está caracterizada por la competencia perfecta.
9. No hay obstáculos impuestos por el gobierno a la actividad económica.

10. Los costos de transporte internos y externos son cero.

11. El análisis se limita a un "mundo" de dos países y dos bienes primarios.

La mayoría de estos supuestos están en el capítulo VII del comercio exterior, por ejemplo, los tres primeros se encuentran en la explicación que Ricardo (1985) hace sobre la regla que regula el valor relativo de las mercancías en un país en específico, y entre dos o más países:

Si los beneficios del capital empleado en Yorkshire fueran superiores a los del utilizado en Londres, el capital se trasladaría rápidamente de Londres a Yorkshire y los beneficios se igualarían; pero si, como consecuencia de la disminución del tipo de producción de la tierra en Inglaterra, debida al aumento del capital y de la población, los salarios vinieran a subir y los beneficios a bajar, no se seguiría de ello que el capital y la población se trasladarán necesariamente de Inglaterra a Holanda, a España o Rusia, países en que los beneficios podrían ser más elevados. (p.139).

Enfatizando en el tercer supuesto, en la teoría del valor-trabajo, los bienes se intercambian en función de las cantidades relativas de trabajo que representan, como ilustra el conocido ejemplo de la cacería de Smith (1983): "Si entre una nación de cazadores, por ejemplo, usualmente cuesta dos veces más trabajo matar un castor de lo que cuesta matar un ciervo, un castor debe, naturalmente, intercambiarse o valer dos ciervos" (p.94).

Con respecto al cuarto supuesto, este se encuentra en los apartados en que Ricardo (1985) analiza el valor del dinero, su poder adquisitivo y los precios (y sus fluctuaciones):

Los beneficios, no nos cansaremos de repetirlo, dependen de los salarios, pero de los salarios reales, no de los nominales; no del número de libras que se paguen anualmente al trabajador, sino del número de días de trabajo necesarios para obtener esas libras. (p.147).

Ricardo (1985) considera fijo el capital al momento de establecer la ventaja comparativa entre dos países, así lo demuestra cuando expresa que, "En un mismo país, puede decirse, 
hablando en términos generales, que los beneficios están siempre al mismo nivel, o difieren solamente en cuanto el empleo del capital pueda ser más o menos seguro y agradable” (p. 139). Además, Ricardo (1985) señala que:

Ahora, supongamos que Inglaterra descubriese un procedimiento especial para elaborar el vino, y que le resultase más ventajoso producirlo que importarlo; naturalmente, trasladaría una parte de su capital del comercio exterior al interior; dejaría de manufacturar tejido para la exportación y produciría vino para su consumo. (p. 142).

A pesar de que el capital es fijo al momento de demostrar la existencia de renta, Ricardo expresa que al momento de eliminar las restricciones a las importaciones, el crecimiento económico se da por la mayor acumulación de capital -que es causa de las Riquezas de la Naciones-, en una economía sin renta. Cuando se plantea como supuesto que los costos unitarios de producción son constantes es porque se afirma lo siguiente: "Así pues, si los salarios siguieran siendo los mismos, los beneficios de los industriales seguirían también siendo los mismos; pero sí, como es seguro, los salarios subieran debido al alza del precio del trigo, sus beneficios disminuirían necesariamente" (Ricardo, 1985, p.114).

Más adelante asevera que:

"En el curso de esta obra he tratado de demostrar que el tipo de beneficios sólo puede ser aumentado por una baja de salarios y que solo puede producirse de modo permanente como consecuencia de una baja en el precio de los artículos de primera necesidad" (Ricardo, 1985, p.137).

Landreth y Colander (2002) explican que la Teoría Malthusiana de la población se empleó para explicar el nivel de los salarios reales a largo plazo. No obstante, las explicaciones ricardianas a corto plazo de los salarios se basaban en un análisis de la oferta y de la demanda de trabajo denominado Doctrina del Fondo Salarial, la que explica los supuestos seis y siete. Éstas no son de verdad curvas de oferta-demanda, tal y como se utilizan en la economía moderna. La demanda de trabajo está determinada por el tamaño del fondo salarial, que es aquella parte del capital acumulado destinada a pagar el trabajo.
Ekelund y Hébert (1992) explican que la doctrina sostiene que los trabajadores dependen de los capitalistas para que éstos les provean de las herramientas necesarias para el trabajo y de los alimentos, vestido y abrigo (es decir, los bienes salariales de subsistencia), a fin de que puedan sobrevivir. La única manera de aumentar el stock de bienes salariales consiste en inducir a los capitalistas a que ahorren, y el único modo de hacer esto es aumentar los beneficios, que, en opinión de Smith, constituyen la única fuente de ahorro.

Siguiendo con Smith (1983), los ahorros tienen que encontrar una salida en el proceso de producción: si se usan para contratar más trabajadores, el fondo de salarios crece, y lo mismo sucede con los pagos (medios) a los trabajadores. En consecuencia, los trabajadores gastan más en bienes salariales, aumenta la demanda agregada y se produce más en el siguiente período de producción $^{1}$. Se supone que todos los ahorros se invierten, dado que el atesoramiento se considera irracional, destinándose al fondo de salarios².

No obstante, Ricardo (1985) propone que el salario siempre marcará una tendencia hacia lo estrictamente necesario, permitiendo al obrero solamente subsistir y reproducirse. A esta propuesta teórica se la identifica como la "Ley de hierro de los salarios". Lo "estrictamente necesario" significaba una baja en los salarios nominales, más no en los reales. Como se citó anteriormente, "la baja de los salarios sólo puede venir por medio de una reducción de los precios de los bienes de primera necesidad".

La interrogante que se presenta es: ¿En base a qué, economista clásico justifica y hace posible una reducción de los salarios nominales, manteniendo los salarios reales? Una primera parte de la respuesta está en el incentivo del empresario hacia la acumulación del capital:

El agricultor y el industrial no pueden vivir sin beneficios, del mismo modo que el trabajador no puede vivir sin salarios. El incentivo para la acumulación disminuirá con cada reducción de los beneficios, y cesará del todo cuando éstos no sean suficientes para constituir una compensación adecuada de las energías desplegadas y del riesgo que ha

1 En este sistema es importante advertir que el dinero se considera solamente como un medio de cambio, no como un depósito de valor.

2 De aquí se deriva lo que se conocerá tiempo después como Ley de Say. 
de correr necesariamente el capital (Ricardo, 1985, p.126).

Si Ricardo (1985) justifica la baja de los salarios para incentivar la acumulación y el crecimiento de la riqueza de un país por medio del aumento de los beneficios del empresario, ¿Cómo es realizable la baja de los salarios nominales? En la liberalización comercial está la respuesta, pero con una condición bastante sui generis según afirma Ricardo (1985):

Si en lugar de cultivar nosotros mismos el trigo, de manufacturar los vestidos y otros artículos de primera necesidad, descubrimos un nuevo mercado que pueda suministrarnos estas mercancías a un precio menor, los salarios bajarán y los beneficios subirán; pero si las mercancías obtenidas a un precio inferior, debido al desarrollo del comercio exterior o a mejoras introducidas en la maquinaria, son solamente las consumidas por los ricos, ningún cambio tendrá lugar en el tipo de beneficios. El comercio exterior, si bien es muy beneficioso para un país, toda vez que aumenta la cantidad y variedad de los objetos en que puedan gastarse las rentas y proporciona incentivos al ahorro y a la acumulación del capital, por la abundancia y baratura de las mercancías, no tiende a hacer subir los beneficios del capital, a menos que los artículos importados sean de primera necesidad para el trabajador. (p.137-138).

El objetivo de Ricardo, en esta parte, es bastante claro: aumentar la tasa de ganancia para quien asume el mayor riesgo en la producción de un determinado bien, ya que dispone su ahorro para el pago del alquiler de la tierra del terrateniente y el pago del salario al trabajador. El trabajador al vender su fuerza de trabajo y el terrateniente al alquilar la tierra, el riesgo es considerado nulo. Recompensar al empresario por medio una economía sin renta y con una alta y creciente tasa de beneficio, es la vía para generar el rápido aumento de la riqueza de un país (Ricardo, 1985).

El octavo supuesto de la economía Ricardiana es la competencia perfecta. Este supuesto se vislumbra de forma bastante explícita cuando los productores de los otros sectores de la economía se ven atraídos por el alto y creciente beneficio del bien agrícola, comportamiento sostenido por el aumento de los precios, y a su vez, del aumento de la población.
Cuando estos productores están atraídos por la alta tasa de rentabilidad del sector agrícola, desplazan sus inversiones hacia este sector haciendo que, con las pretensiones de los terratenientes de aumentar su renta, las ganancias del productor agrícola disminuyan de ese punto tan alto. Aquí es donde opera uno de los supuestos más importante de la competencia perfecta: la libre entrada y salida de empresas.

La formación de precios está en manos de muchos productores y muchos compradores, así que cuando entran más productores, sumado a otros factores propios de la dinámica de la teoría Ricardiana, las ganancias del productor de cierto bien agrícola, como el trigo, tiende a la baja después de ver aumentado sostenidamente por el aumento de la demanda. Ricardo (1985) lo expresa así de la siguiente manera: "En un sistema de comercio perfectamente libre, cada país, naturalmente, dedica su capital y trabajo a los empleos que le son más beneficiosos. Esta tendencia a la ventaja individual está admirablemente relacionada con el bien universal del mundo" (p.138).

Cuando se trata de la mercancía universal Ricardo (1985) expresa que:

El oro y la plata, que han sido escogidos como instrumento general de la circulación, están -por efecto de la competencia comercialdistribuidos entre los distintos países del mundo en forma tal que se ajustan al tráfico natural que tendría lugar si esos metales no existieran y el comercio entre las naciones fuera puramente de permuta. (p.147).

Y cuando se trata del mercado de trabajo Ricardo (1985) afirma que:

Estas son, pues, las leyes por las cuales se regulan los salarios y se rige la felicidad de la mayoría de los componentes de toda comunidad. Como todos los demás contratos, el ajuste de los salarios debe dejarse a la libre concurrencia del mercado y no debe nunca ser controlado por medio de leyes. (p.147).

El último de los supuestos que trataremos en este trabajo es el nueve ${ }^{3}$, donde Ricardo (1985) hace imprescindible la no presencia del Estado en la economía, ya que solo debe estar para lo más indispensable: fundamentos Smithianos. Cuando la teoría Ricardiana expresa que no hay

3 Los supuestos diez y once no se tratarán por considerarse los más elementales de la teoría. 
obstáculos impuestos por el gobierno a la actividad económica, lo hace para el mercado laboral, el intercambio comercial y la circulación de oro. "La tendencia perniciosa de estas leyes no es un misterio, desde que ha sido plenamente desarrollada por la hábil pluma de Mr. Malthus; y todos sus amigos de los pobres deben desear ardientemente su abolición” (Ricardo, 1985, p.109).

\section{LA PROPUESTA DE LIBRE COMERCIO}

Hasta acá se ha explicado cada supuesto con el objetivo de hacer más comprensible el modelo y su justificación hacia el libre comercio, por medio de la ventaja comparativa. No obstante, ¿Cuál es el contexto en el que se desarrolla la teoría expuesta por David Ricardo?: la controversia sobre las leyes de Cranos, que se suscitó durante las guerras napoleónicas; el embargo de Napoleón sobre los puertos británicos que impidió la entrada de los granos extranjeros; y los agricultores británicos se vieron obligados a aumentar la producción de cereal doméstico, a fin de alimentar a la población.

Sin embargo, los costos de producción eran altos en Inglaterra en comparación con el resto del mundo, aumentando el precio del cereal británico: entre 1790 y 1810 el aumento en promedio fue del $18 \%$ anual. Ese incremento fue provocado por el crecimiento de la población, variable que se vuelve imprescindible en lo expuesto por Ricardo (1985), "A cada nuevo incremento de la población que obligue a un país a recurrir a los terrenos de menor fertilidad para poder procurarse los alimentos necesarios, la renta sobre los terrenos más fértiles irá subiendo" (p.72).

La expansión de la demanda, provocado por el aumento de la población, descubre la insuficiencia de Inglaterra con respecto a la cantidad de tierras fértiles, factor productivo que es abundante en otros países por su extensión territorial y condiciones naturales. No obstante, la anterior cita también refleja la llamada renta diferencial. Con el aumento de los precios, las rentas de la tierra también aumentaron, hasta el punto de que los terratenientes desarrollaron unos intereses creados para continuar restringiendo las importaciones de cereales. Las Leyes de Granos aprobadas por el parlamento en 1815 hacían posible estos intereses.

Ricardo observa que Inglaterra tiene una cantidad limitada de tierras muy fértiles (alta productividad) y otras de tierras poco fértiles (baja productividad), señalando que el valor que cobran los terratenientes será mayor si la tierra tiene una productividad alta. ¿Pero qué incentiva al terrateniente a cobrar una renta más alta? Todos los terratenientes pedirán una renta más alta, unos más que otros, pero todos exigirán un aumento. La respuesta está en el aumento de la tasa de ganancia del productor, y el mayor número de éstos que hacen presión sobre el valor de la tierra.

A medida que los precios suben, en un primer escenario, los productores se ven beneficiados, con lo que su tasa de ganancia aumenta incentivando a los terratenientes a pedir un aumento de su renta, y a los productores de los otros sectores económicos a invertir sus capitales en la producción agrícola (o un determinado producto agrícola, por ejemplo, el trigo). Aquí se ven dos supuestos anteriormente explicados: competencia perfecta, y los factores productivos son móviles dentro de un país.

La atracción que genera una mayor tasa de ganancia para los productores hace que esta se reduzca en un posterior escenario: el aumento de la renta, la baja productividad de las tierras poco fértiles, la escasez de las más fértiles, y una mayor cantidad de productores de otros sectores económicos, son factores que llevan a la tasa de ganancia a disminuir. Ricardo (1985), ante esto, expresa que la acumulación disminuirá con cada reducción de los beneficios.

La preocupación de Ricardo está en que una renta mayor para el terrateniente hará que la acumulación no sea posible. El progreso de la renta debe ser lento. De aquí viene, una de sus frases más célebres, "El trigo no es caro porque se paga renta, sino a la inversa: se paga renta porque el trigo es caro" (Ricardo, 1985, p.79).

Es así, que Ricardo (1985) después de tratar el tema de la renta diferencial, los salarios, y las ganancias de los productores, argumenta a favor del libre comercio para que el trigo (materia prima) deje de ser costoso:

Si Portugal no tuviera relaciones comerciales con otros países, en lugar de emplear gran parte de su capital e industria en la producción de vinos, con los cuales adquiere para su uso los tejidos y artículos manufacturados de otros países, se vería obligado a destinar una parte de su capital a la manufactura de esos artículos, que obtendría, probablemente, en calidad y en cantidad inferiores.(p.139).

En base a la ventaja comparativa, argumenta Ricardo (1985) sobre en qué condiciones se hace posible la acumulación del capital: 
Por grande que sea un país, si la tierra es de mala calidad y la importación de alimentos está prohibida, las acumulaciones más moderadas de capital irán acompañadas de grandes reducciones en el tipo de beneficios y de un aumento rápido de la renta, y, por el contrario, un país pequeño pero fértil, especialmente si permite libremente la importación de alimentos, puede acumular una gran cantidad de capital sin gran disminución en el tipo de beneficios, ni gran aumento de la renta de la tierra. (p.131).

En un subsiguiente escenario, con la eliminación de barreras arancelarias, la renta de la tierra desaparece y la tasa de la ganancia del productor aumenta. Ricardo argumenta que el objetivo es que exista una compensación adecuada de las energías desplegadas y del riesgo que ha de correr necesariamente el capital invertido.

Pero hay que precisar. Ricardo no solo busca el aumento de la tasa de ganancia por medio de la desaparición de la renta a través del libre comercio (por ejemplo, un trigo más barato y de más calidad), sino también a través de una rebaja de los salarios nominales, más no de los reales. Esos salarios pertenecen a la llamada "Ley de Hierro".

Esta rebaja es solo posible si el libre comercio beneficia a la clase trabajadora. Al fin de cuentas, el poder adquisitivo no cambia. Una caída de los precios de primera necesidad por el libre comercio y una rebaja de salarios nominales para aumentar la tasa de ganancia de los productores, hacen cumplir la "Ley de Hierro".

El objetivo final de Ricardo en su obra es justificar el libre comercio en base a la división internacional del trabajo, expuesto anteriormente por Smith (1983). Para ello, el contexto en que se desarrolla la propuesta, la lucha de clases entre terratenientes y productores, la Riqueza de las Naciones de Adam Smith, y las condiciones naturales de Inglaterra, su posición y condición monocentrista en el mundo, hacen posible tal propuesta.

\section{CONCLUSIONES}

Es indudable que Adam Smith en su obra Las Riquezas de las Naciones le da un vuelco a la política económica imperante en el siglo xviII. Pero la obra de Ricardo goza de una trascendencia aún mayor, poco reconocida, porque demuestra la pugna entre dos clases sociales, y cómo se construye teóricamente la economía clásica desde esta pugna. Ricardo observa un problema que está compuesto por el aumento de la población y de los precios, la lucha por el excedente, y principalmente, por las limitaciones que tiene Inglaterra para acumular capital con mayor rapidez.

Ricardo al analizar este problema mira al comercio exterior como una vía para resolverlo, y realiza una propuesta para cambiar el comercio internacional en un 'juego' donde todos salgan ganando. Le llama poderosamente la atención la forma en cómo el productor obtiene su tasa de ganancia y los obstáculos para aumentarla. Encuentra que la renta es ese obstáculo y que su crecimiento no solo obstaculiza la acumulación del capital, sino que lo contrae.

El comercio exterior se convierte en una oportunidad para quien arriesga más, y un sendero por donde Inglaterra pueda expandir su Frontera de Posibilidades de Producción (FPP), siendo solo lo que es, una isla.

\section{REFERENCIAS BIBLIOGRÁFICAS}

Appleyard, D. y Field, A. (2003). Economía Internacional (4a ed.). Bogotá, Colombia: McGraw-Hill.

Ekelund, R. B., Y Hébert, R. F. (1992). Historia de la teoría económica y de su método (Tercera Edición). Madrid, España: McGraw Hill.

Krugman, P. y Obstfeld, M. (2006).Economía Internacional, Teoría y política (7a ed.). Madrid, España: Pearson Educación.

Landreth, H. y Colander, D. (2002).Historia del pensamiento económico ( $1^{\underline{a}}$ ed. en español). México D. F.: CECSA.

Ricardo, D. (1985).Principios de economía política y tributación. Barcelona, España: Ediciones ORBIS.

Smith, A. (1983). Una investigación sobre la naturaleza y causa de la riqueza de las naciones. Barcelona, España: Ediciones ORBIS.

Tugores, J. (2006).Economía Internacional, Globalización e integración regional ( $6^{\mathrm{a}}$ ed.). Madrid, España: McGraw-Hill. 